\title{
A Technique for Closed Interlocking Nailing of Midshaft Tibial Fractures without Image Intensification
}

\author{
${ }^{1}$ Manoj Mathew, ${ }^{2}$ Mandeep S Dhillon, ${ }^{3}$ Harinder Gakhar
}

\section{ABSTRACT}

Objective: In this study, we evaluated the reliability, reproducibility and final outcome of our techniques for performing image independent tibial interlocking nailing

Design: Prospective.

Setting: Tertiary teaching hospital (level I trauma center).

Patients/participants: A total of 29 patients were selected for the study (21 males and 8 females patients).

Intervention: Locked tibial intramedullary nailings were performed in the selected subjects, using the standard AO interlocking nail (Synthes ${ }^{\mathrm{TM}}$, Switzerland), utilizing our improvisations of the standard technique.

Main outcome measurements: The successful completion of the technique was the prime parameter evaluated. Total surgical time, time taken for placement of each distal bolt, fluoroscopy time (when used) and postoperative alignment and reduction were evaluated. The patients were followed at 4 weekly intervals.

Results: The average duration of the surgery was 78.6 minutes. The mean distal locking time was $16.2 \pm 1.4$ minutes. Fluoroscopy had to be used in three cases, when the distal locking by the 'nail-over-nail' technique failed. In one case, the intramedullary position of the guidewire had to be verified. Thus, 26 out of $30(86.7 \%)$ cases were performed without any imaging assistance. In two cases, the fracture site had to be opened after difficulties were encountered in achieving closed reduction. Thus, 24 out of 30 procedures $(80 \%)$ were successfully completed by our technique. The fractures were followed to union and the long-term results were comparable to the results of tibial fracture operated by image assistance.

Conclusion: The technique described here can come handy in situations of image intensifier failure. Routine use of part of the steps can assist in reducing radiation exposure during interlocking nailings.

Keywords: Fracture fixation, Intramedullary nailing, Tibial fractures, Image intensification, Distal locking, Image independent nailing.

\footnotetext{
${ }^{1}$ Registrar, ${ }^{2}$ Professor, ${ }^{3}$ Senior Spinal Fellow

${ }^{1}$ Department of Orthopedics, Royal Brisbane and Women's Hospital and Royal Children's Hospital, Queensland, Australia

${ }^{2}$ Department of Orthopedics, Postgraduate Institute of Medical Education and Research, Chandigarh, India

${ }^{3}$ Department of Orthopedics, Royal Derby Hospital, United Kingdom

Corresponding Author: Manoj Mathew, Registrar, Department of Orthopedics, Royal Brisbane and Women's Hospital and Royal Children's Hospital, Queensland, Australia, Phone: 61736368111, e-mail: drmanoj4u@yahoo.com
}

How to cite this article: Mathew M, Dhillon MS, Gakhar H. A Technique for Closed Interlocking Nailing of Midshaft Tibial Fractures without Image Intensification. J Postgrad Med Edu Res 2014;48(3):111-116.

Source of support: Nil

Conflict of interest: None

\section{INTRODUCTION}

The standard procedure for locked intramedullary nailing of the tibia utilizes fluoroscopic imaging and radiographic exposure. The ability to perform a closed interlocking nailing of tibia fractures without image intensification offers two main benefits which are as follows:

1. The ability to complete a closed interlocking nailing of the tibia in the event of failure or malfunction of the available image intensifiers,

2. Utilize components or all of the steps in the below described technique to minimize radiation exposure.

It is ideal to keep radiation levels to as low as reasonably achievable. Radiation effects are both the deterministic and stochastic. Deterministic effects have a threshold, but on the other hand, the stochastic effects have a cumulative effect over time. ${ }^{1}$ The highest radiation exposure is to the surgeons hands during most orthopedic procedures but it also scatter to the surgeons eyes, thyroids and torso. Most of the exposure in a tibial intramedullary nailing occurs during the introduction of the distal bolts. Several devices have been used to achieve distal targeting of the locking bolts without fluoroscopy, including proximally mounted mechanical jigs,${ }^{2-4}$ laser guided systems ${ }^{5,6}$ navigation based methods ${ }^{7}$ and electromagnetic targeting devices. ${ }^{8}{ }^{8}$ Although these devices have been demonstrated to be successful, the devices used necessitate the procurement of additional inventory with significant added costs. The other major steps commonly requiring image intensification are confirmation of the entry site position, ensuring intramedullary location of the guidewire after fracture reduction, and centralizing the nail distally within the canal.

In the orthopedic trauma unit of Postgraduate Institute of Medical Education and Research (PGIMER), Chandigarh, we developed a set of simple techniques that made it possible to perform an interlocking tibial nailing without the need for image intensification at any stage. The aim of this 
study was to evaluate prospectively, the reliability of these techniques to minimize and potentially, completely negate the necessity of image intensification during interlocking nailing of tibial shaft fractures.

\section{MATERIALS AND METHODS}

A total of 30 consecutive tibial diaphyseal fractures in 29 patients were selected for the study (21 male and 8 female patients). Exclusion criterion included, open fractures, skeletal immaturity, extremely osteoporotic bone, metaphyseal fracture location, and $\mathrm{AO}$ classification type $\mathrm{C}$ fractures. The standard AO interlocking nail was used (Synthes ${ }^{\mathrm{TM}}$, Switzerland) in all cases.

\section{Surgical Technique}

The patient is positioned supine. The entry site is made using an incision extending from the inferior pole of the patella to the tibial tubercle. A transpatellar tendon split was utilized and the entry site is selected. If a transpatellar incision or approach is used, it is noted that the guidewire or awl entry should actually be medialized from the line of this split by about 5 to $7 \mathrm{~mm}$ to gain a midline entry site into the tibia. After passing the awl, a rigid cannulated reamer of diameter $8 \mathrm{~mm}$ was used in the proximal fragment. Once it was felt that the cannulated reamer has crossed, the proximal fragment it is withdrawn slightly. With the knee in flexion, traction is applied downwards along the axis of the tibial shaft so as to achieve approximate reduction. The reduction is palpated and the cannulated reamer is now advanced with gentle turns. Unlike a guidewire, which tends to slip out through the edges of unreduced segments of bone, the larger diameter of the reamer makes it grasp the distal fragment from within and internally align it into reduction. The cannulated reamer should be advanced gently past the fracture

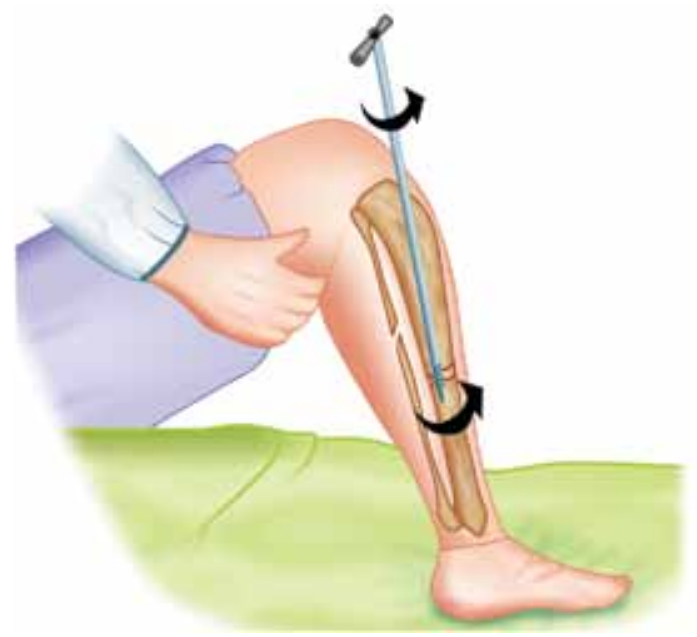

Fig. 1: The rigid cannulated $8 \mathrm{~mm}$ reamer is passed into the distal fragment with gentle turns. The immobility at the fracture site and the palpable torque in the distal fragment are evidence of the intramedullary location
(Fig. 1). This step has to be done gently and cautiously to avoid completion of an unseen communition. Several current nailing systems do have a reduction tool that is essentially a cannulated solid rod with a flared tip intended for guidewire redirection. The reduction tool can be used in place of the reamer. The guidewire is now inserted into the cannula of the reamer (Fig. 2). If the reamer is intramedullary, the grating sensation of the guidewire passing through the cancellous bone and subsequent 'thud' of the guidewire hitting the plafond can be felt.

Further, reaming is done in the usual manner using sequential flexible reamers (Fig. 3). The reaming is done so as to ensure that the largest possible nail is used. Using the largest nail possible is critical to the technique, because it ensures centering of the nail distally.

Once the nail is introduced, the distal locking is performed. The knee is flexed to more than $90^{\circ}$ and the limb placed in a figure of four position. A nail of same length is mounted on the medial aspect of the tibia under the insertion handle. The tissue protection sleeves with trocar are placed in the slots provided in the insertion handle and then through the proximal holes of the outer nail (Fig. 4). This ensures that the extramedullary nail is placed on the medial aspect of the tibia, and aligned approximately parallel to the intramedullary nail. The distal part of the extramedullary nail is centered on the medial aspect of the distal tibia (Fig. 4).

The drill hole for the distal most bolt is made first. The distal most hole in the extramedullary nail is used as a guide for location of the level of the holes for the intramedullary nail. The angular orientation of the sleeve trocar assembly in the insertion handle is used as a visual guide to the angular orientation of the distal bolt holes of the intramedullary nail. Utilizing the distal most hole of the extramedullary nail as a guide for positioning and with the drill bit itself positioned parallel in two planes to the sleeve and trochar assembly, a

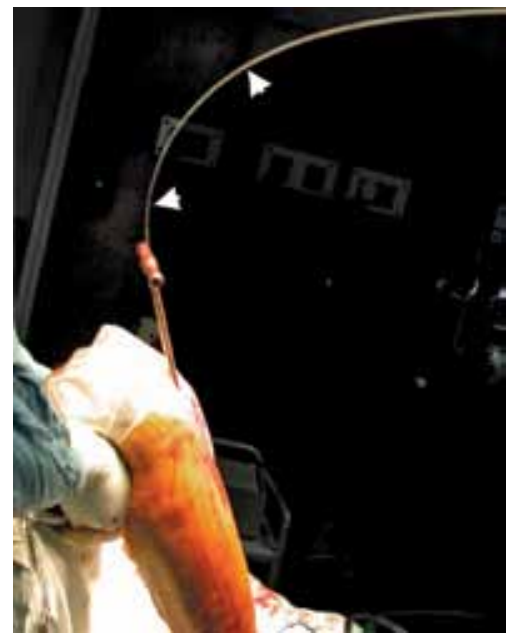

Fig. 2: The guidewire (white arrows) is now inserted into the cannula of the rigid reamer. If the reamer is intramedullary the grating sensation of the guidewire passing through the cancellous bone and subsequent 'thud' of the guidewire hitting the plafond can be felt 


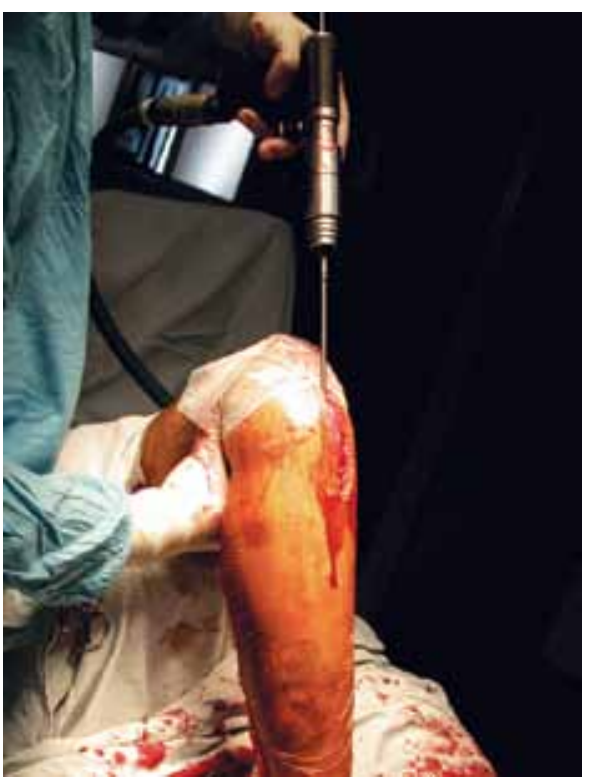

Fig. 3: Sequential reaming using flexible reamers
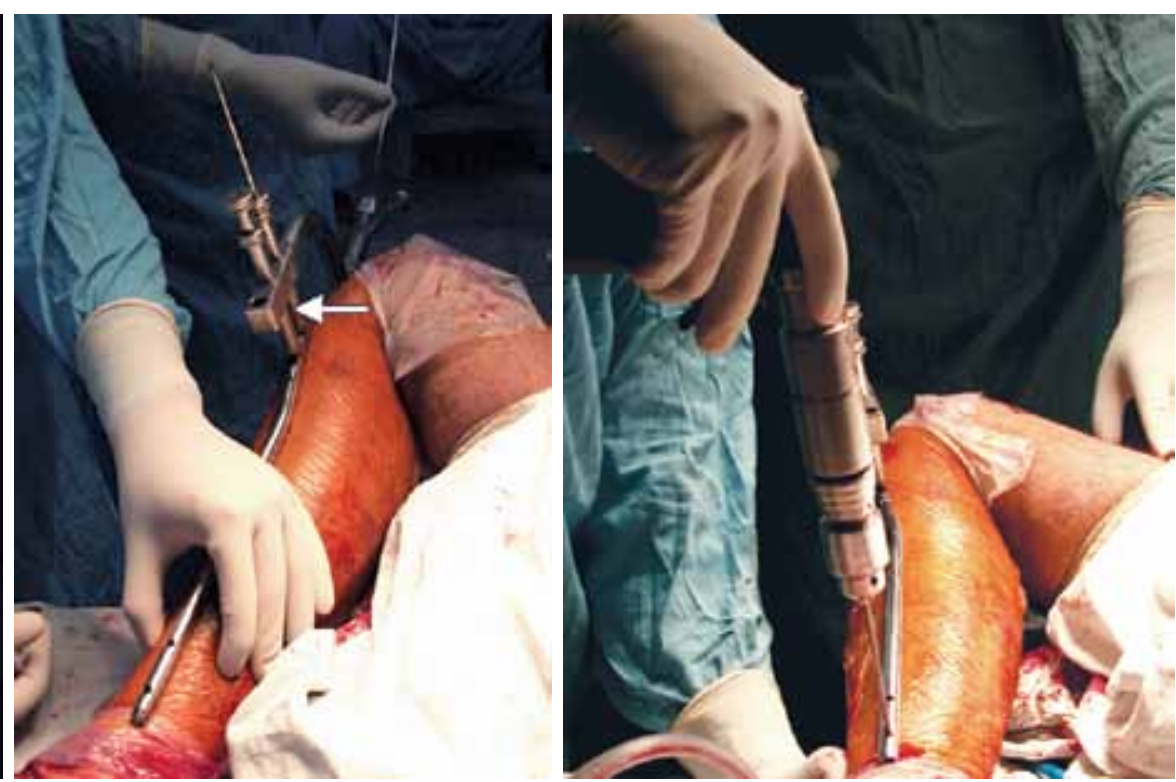

Fig. 4: A nail of same length is mounted Fig. 5: The distalmost hole is made first, using medially. Colinearity of this nail with the the hole in the outer nail as a guide. The drill intramedullary nail is ensured by pla- should be parallel to the trochar and sleeves in cing the sleeves with trochar through both planes the proximal holes (white arrow) and by distally centering of the nail by palpating the anterior and posterior tibial borders

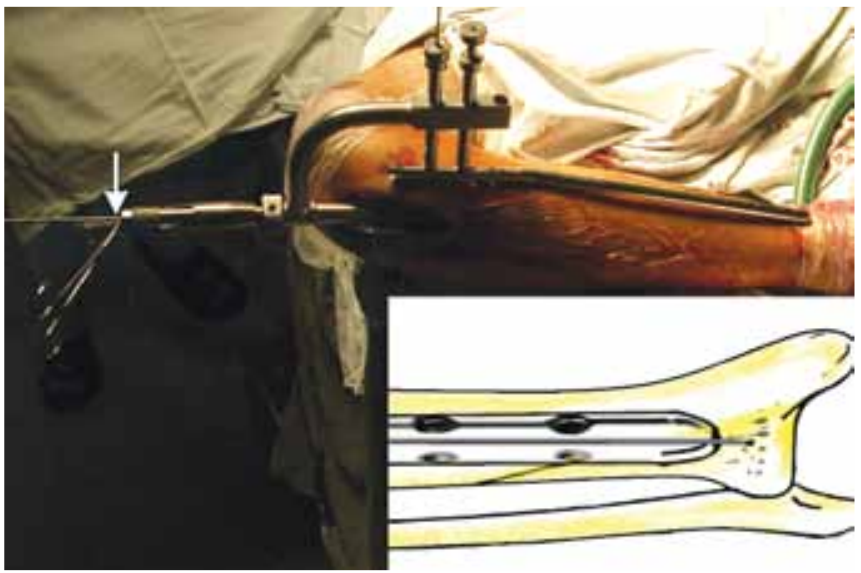

Fig. 6: After the drill is removed the guidewire is fully inserted till it hitches against the plafond. A hemostat is now placed (white arrow) at the point where the guidewire exists the jig. Inset; schematically shows the intramedullary position of the guidewire at this position

unicortical drill hole is now made (Fig. 5). This hole is used as a pilot hole to verify location.

If the pilot drill hole and the intramedullary nails bolt hole are in line, then the accuracy of placement can be checked using the long guidewire in what we shall refer to as a guidewire technique. After the drill is removed, the guidewire is fully inserted till it hitches across the plafond. A hemostat is now placed at the point where the guidewire exists the jig (Fig. 6). The guidewire is withdrawn and the drill is reinserted. If the hemostat stays short of the jig edge by more than $2 \mathrm{~cm}$ and the impact of the guidewire hitting the drill bit is palpable through the drill, then it confirms proper location of the hole (Fig. 7).

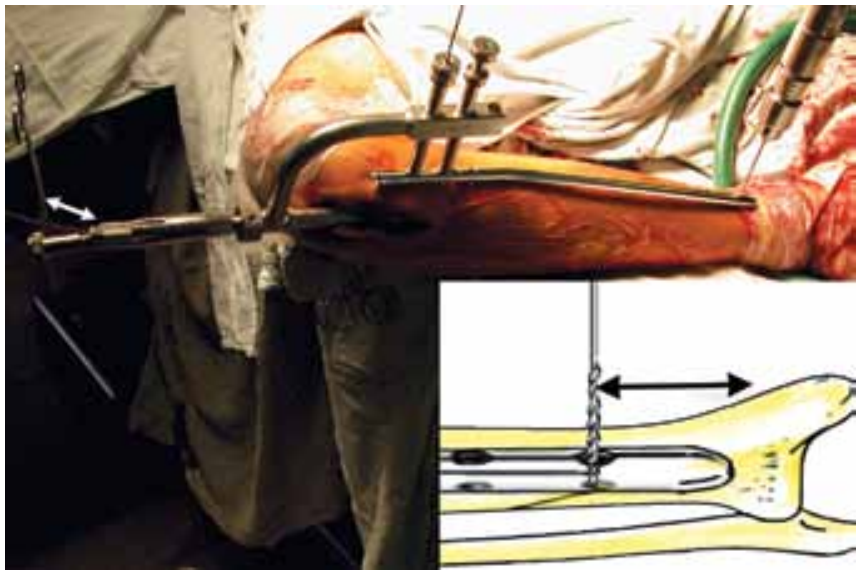

Fig. 7: With the drill bit in situ the guidewire is fully reintroduced. Inset; schematically demonstrates the amount by which the guidewire is restricted by the drill bit (indicated by the black double arrow), this corresponds to the distance measured externally (indicated by the white double arrow)

If by this check the hole placement is found to be erroneous or outside the nail, then a $1 \mathrm{~mm} \mathrm{~K}$-wire is used to feel through the pilot hole. Minor rotational mismatches can be adjusted by slightly rotating the nail using the insertion handle. In case of anterior or posterior translational mismatch or of distal or proximal length mismatch, the amount of mismatch is gauged by using this $\mathrm{K}$-wire. The mismatch is most often to the order of $5 \mathrm{~mm}$ to $1 \mathrm{~cm}$ and is very often identifiable by using the $\mathrm{K}$-wire, introduced through the pilot hole, to walk up and down the nail or anterior and posterior to the hole to find the nail and the bolt hole. Once the bolt hole in the nail is felt, the $\mathrm{K}$-wire is forced in through the bolt hole, often at an angle. The 


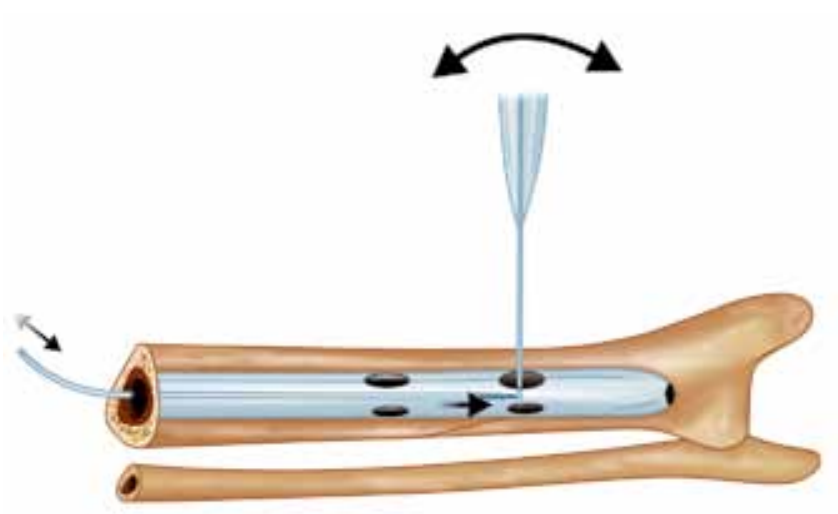

Fig. 8: Schematic illustration of the K-wire method to check the location of the screw hole in the nail. When within the screw hole, the K-wire will rattle to the impact of the guidewire

K-wire's position is now confirmed to be within the bolt hole by striking the guidewire, passed within the nail, against the $\mathrm{K}$-wire. The $\mathrm{K}$-wire will be seen to rattle to each impact of the guidewire if it is indeed within the bolt hole (Fig. 8). If the actual bolt hole is grossly off the mark compared to the pilot hole, then a new drill hole is made at the level of the new presumed point, still utilizing the distal hole in the overlying nail as a gauge of length and angles. Once the drill position is verified to be intramedullary by the above described guidewire technique, the drill hole is completed and the locking bolts are placed. The bolt placement is again confirmed by using the guidewire technique against the bolt. The length at which the guide wire hits the distal bolt can be used as a confirmatory check. This can be done by using the hemostat marking method described above or by taking out the guidewire and placing it outside in close apposition to the medial border of the tibia to confirm that the length at which the bolt has been fixed (from the jig edge to the skin stab wound) is same as the distance at which the guidewire meets resistance when placed inside the nail.

Now, a screwdriver is placed in the screw head of the distal screw so as to act as a reference of its direction. Using the guidance provided by the proximal sleeves, the angle of the screwdriver and the hole in the overlying nail, the hole for the proximal of the distal locking bolts is made. The position is similarly confirmed by sounding the guidewire against the stationary drill bit or by using a K-wire. The second cortex is drilled and bolts placed. After correction of rotational alignment, the nail extractor is used to backslap the distal fragment into compression at the fracture site and proximal locking is performed. In fractures without comminution and in which the obliquity of the fracture line was less than $30^{\circ}$, the proximal locking consisted of only the dynamic bolt. In others, the static bolt was applied as well.

Postoperatively, the patients were encouraged early assisted weight bearing and subsequently unassisted weight bearing.

\section{Clinical and Radiographic Evaluation}

All intraoperative parameters including total surgical time, time taken for placement of each distal bolt, fluoroscopy time (when used), and intraoperative complications were noted. The postoperative radiographs were evaluated for fracture reduction, varus-valgus malalignment, entry site proximity to joint line, nail prominence, and nail centering. Postoperative rotational alignment was evaluated by seating the patient at the edge of the bed with the knees in flexion.

All patients were evaluated at monthly intervals. After union was documented, further follow-ups were conducted at three monthly intervals. Radiographs were also taken to evaluate for union and to look for evidence of malalignment.

Malunion was defined as $>5^{\circ}$ of angulation on anteroposterior or lateral radiographs or rotational malalignment $>15^{\circ}$. Clinical union was defined as the ability to bear full weight with no pain at the site of the fracture, and radiographic union was defined as evidence of bridging of three of the four cortices on standard anteroposterior and lateral radiographs. ${ }^{10}$ Union occurring after 20 weeks was classified as delayed union. ${ }^{11}$ Nonunion was defined as cessation of periosteal and endosteal new bone formation, with sclerosis of the medullary canal observed at the fracture surfaces. ${ }^{12}$ The patient was evaluated clinically for pain (at the fracture site, at the nail entry site and the sites of the locking bolts) and for function. Anterior knee pain was registered on a visual analog scale ranging from 0 to 10 with 10 as the worst.

\section{RESULTS}

A total of 30 consecutive tibial diaphyseal fractures in 29 patients were selected for the study (21 male and 8 female patients). Two patients were lost to follow-up. In two cases, the fracture site had to be opened after difficulties were encountered in achieving closed reduction. The average duration of the surgery was 78.6 minutes (54-118). The mean distal locking time was $16.2 \pm 1.4$ minutes (9-38 minutes). Fluoroscopy had to be used in three cases when the distal locking by the nail-over-nail technique failed. In one case, the intramedullary position of the guidewire had to be verified (fluoroscopy time of 12 seconds). Barring these four instances fluoroscopy was not used in any of the other 26 nails.

In one case, there was malrotation greater than $15^{\circ}$. In this patient, a second surgery was performed; wherein, the distal bolts were removed, the fracture realigned and then relocked. In two cases, the drill bit broke while drilling the proximal of the two distal holes.

The mean period of follow-up was 12 months (6-24 months). The mean time taken to union was 16.8 weeks 
$(10-32$ weeks, $S D=4.5)$. In four patients, the nails had to be dynamized. Delayed union had occurred in two cases of which one case required bone-grafting. No case of nonunion occurred in the series. No cases of nail failure was noted over the follow-up period. One case of bolt fatigue did occur.

Anterior knee pain of varying intensity was noted in 14 cases (46.7\%; median visual analog score 5). Although there was a significant relief of knee pain in the cases in which the nail was removed ( 6 cases; all cases with a visual analog score more than 6), some amount of knee discomfort did persist in three of these patients (median score of 2 on visual analog scale). Four patients ( $7.5 \%)$ had pain and tenderness in the vicinity of the distal locking site. In three of these cases, the distal locking bolts were longer than what should have been the ideal length. In all cases, this pain settled after the distal bolts were removed.

\section{DISCUSSION}

Over the past decade it has come to be accepted that, in tibial diphyseal fractures, intramedullary nails are more advantageous for fracture healing and function than other available methods of treatment like casting and plating..$^{10}$ The standard procedure for tibial interlocking is fluoroscopy dependent. Recent concerns of radiation exposure to the surgeon and patient has been one of the main reasons for the development of methods that attempt to minimize radiation exposure while performing a tibial interlocking. ${ }^{4-7,11-13}$ Skjeldal and Backe in 1987 reported that exposure to 5 minutes fluoroscopy during distal interlocking will limit the number of operations performable, before the maximum permissible radiation dose for the hand is reached, to 25 per year if the hand is placed in the primary X-ray beam. ${ }^{14}$ They recommend using an awl with $30 \mathrm{~cm}$ long side arm for distal locking. Madan and Blakeaway conducted a study, where they estimated the radiation dosage to the surgeons extremities and patients gonads. ${ }^{15}$ Although they conclude that the exposures using the current techniques are well within acceptable limits, they also caution that since the long-term effects of radiation are unknown and because establishing a causal relationship to many types of cancer is difficult; it would be justifiable to minimize exposure and take all the necessary precautions. Mehlmann and DiPasquale demonstrated that operating room personnel within 24" from the fluoroscopy beam receive significant amounts of radiation exposure, particularly to the unprotected eyes, neck and hands. ${ }^{16}$

In the developing world quite a sizeable number of primary and secondary hospitals do not have C-arms, and in the ones where they are available technical problems and breakdowns are not infrequent. It is the latter situation that made us chance upon and further develop techniques to do closed tibial interlocking without image intensification. In this study, we have evaluated the reproducibility and efficacy of these techniques and followed the fractures till union. Our study uses nothing more than standard interlocking equipment for performing all the steps (rather than distal locking alone) in tibial interlocking without image intensification.

In our study, 26 out of 30 ( $86.7 \%$ ) cases were performed without any imaging assistance. One of the steps requiring fluoroscopic assistance is achieving reduction. In all except 3 cases closed reduction and guidewire placement was successful, out of which one failed case required fluoroscopic assistance for verification of the guidewire position and the other two had to be opened, because the reamer encountered resistance in passing it into the distal fragment. This translates to a $90 \%$ success rate for the cannulated reamer assisted closed reduction technique. If the two cases in which the fracture site was opened are considered as failures of the technique, then 24 out of 30 procedures $(80 \%)$ were successfully reduced closed by our technique, without any imaging or necessity to open the fractures.

The entry site was made under direct vision to minimize cartilage and soft tissue damage that could possibly contribute to anterior knee pain. The incidence of anterior knee pain was however still substantial at $46.7 \%$, but this is similar to the results reported in other studies. ${ }^{17,18}$

In 27 out of 30 cases $(90 \%)$ distal locking was done without image guidance. The average distal locking time was $16.2 \pm 1.4$ minutes with a range, however, of 9 to 38 minutes. The longer cases were done during our initial learning curve phase. A probability plot of distal interlocking times (Fig. 9) shows that $80 \%$ cases were locked within 16 minutes (this includes time taken to drive in the screws and countercheck their position by sounding the guidewire). It is our consensus that if the distal hole cannot be located and locked even after 20 minutes by this technique then one should resort to image assisted locking, if available. In two cases, the drill

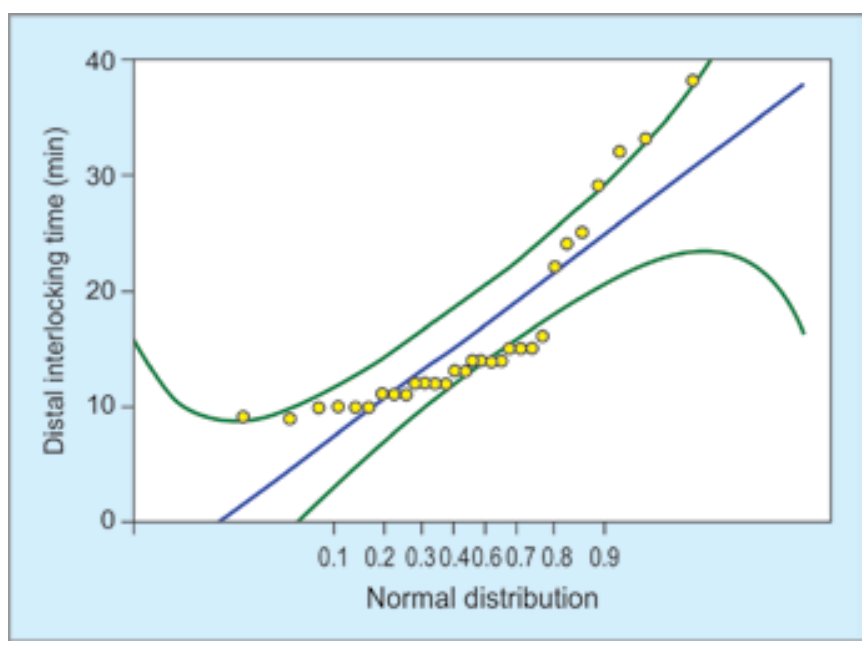

Fig. 9: Probability plot of distal locking time 
bits broke. This was due to excessive leverage force applied over the drill bit in an attempt to negotiate it from an eccentric entry hole into the opposite side of the hole in the nail. When this technique is to be used, drilling has to be gentle and caution should be used to avoid excessively bending the drill bit to negotiate it around an eccentrically placed entry hole. Always ensure that the guidewire is withdrawn while the drill is moving.

The time taken to union in our series is comparable to other studies of tibial fracture union after nailing. ${ }^{19}$ At the outset of the study, we were wary of the possibility of malalignment, improper positioned bolts, the possibility of early bolt fatigue (because of possible eccentricities in bolt placement), and vascular injury due to the 'blind' nature of the technique. Except for one case of bolt fatigue and one case of excessive malrotation none of the other complications occurred. One complication noted in our series was pain at the distal bolt site which occurred in 4 patients (7.5\%). This however settled after the bolts were removed. There were no delayed presentations of malrotation in our series indicating that the effective rotational locking provided was sufficient. In addition to excessive bolt lengths in three of the four cases, residual micromotion at the distal locking bolt site due to a wider entry hole (where a coalescent adjacent hole had to be placed) is a possible cause for the $7.5 \%$ incidence of pain at the distal bolts reported in our series.

It is not advisable to use the techniques in fractures close to the metaphyseal region because of the much higher chance of malalignment. In severe degrees of communition in patients with tighter medullary canals a blind reamer assisted reduction technique has the potential to cause more communition. We believe that reduction in those situations should always be image assisted. In cases of severe swelling and obesity, palpation of the medial tibial crest and verification of the quality of the reduction may be difficult. Multiple failed blind drill holes and coaleascent entry site drill holes do have the theoretical potential to cause incomplete locking. A patient and step-wise approach is needed to avoid this. By the end of our learning curve locking was possible without needing more than two holes in most cases.

Despite its drawbacks, we believe that knowledge of the above described technique could come in handy when the image intensifier fails you, and parts of the technique could be used as technical tips to overall reduction of radiation exposure.

\section{REFERENCES}

1. Singer G. Occupational radiation exposure to the surgeon. J Am Acad Orthop Surg 2005;13(1):69-76.
2. Krettek C, Konemann B, Miclau T, Kolbli R, Machreich T, Kromm A, Tscherne H. A new mechanical aiming device for the placement of distal interlocking screws in femoral nails. Arch Orthop Trauma Surg 1998;117(3):147-152.

3. Pardiwala D, Prabhu V, Dudhniwala G, Katre R. The AO distal locking aiming device: an evaluation of efficacy and learning curve. Injury 2001;32(9):713-718.

4. Pennig D, Oppenheim W, Faccioli G, Rossi S. Intramedullary locked nailing of femur and tibia: insertion of distal locking screws without image intensifier. Injury 1997;28(4):323-326.

5. Goodall JD. An image intensifier laser guidance system for the distal locking of an intramedullary nail. Injury 1991;22(4):339.

6. Goulet JA, Londy F, Saltzman CL, Matthews LS. Interlocking intramedullary nails: an improved method of screw placement combining image intensification and laser light. Clin Orthop Relat Res 1992;281:199-203.

7. Yaniv Z, Joskowicz L. Precise robot-assisted guide positioning for distal locking of intramedullary nails. IEEE Trans Med Imaging 2005;24(5):624-635.

8. Hoffmann M, Schroder M, Lehmann W, Kammal M, Rueger JM, Herrman Ruecker A. Next generation distal locking for intramedullary nails using an electromagnetic X-ray-radiation-free realtime navigation system. J Trauma Acute Care Surg 2012;73(1): 243-248.

9. Stathopoulos I, Karampinas P, Evangelopoulos DS, Lampropoulou-Adamidou K, Vlamis J. Radiation-free distal locking of intramedullary nails: evaluation of a new electromagnetic computer-assisted guidance system. Injury 2013;44(6):872-875.

10. Schmidt AH, Finkemeier CG, Tornetta P 3rd. Treatment of closed tibial fractures. Instr Course Lect 2003;52:607-622.

11. Gugala Z, Nana A, Lindsey RW. Tibial intramedullary nail distal interlocking screw placement: comparison of the free-hand vs distally-based targeting device techniques. Injury 2001;32: SD21-SD25.

12. Hudson I. Locking nailing: an aid to distal targetting. Injury 1989;20(3):129-130.

13. Suhm N, Messmer P, Zuna I, Jacob LA, Regazzoni P. Fluoroscopic guidance vs surgical navigation for distal locking of intramedullary implants. A prospective, controlled clinical study. Injury 2004;35(6):567-574.

14. Skjeldal S, Backe S. Interlocking medullary nails-radiation doses in distal targeting. Arch Orthop Trauma Surg 1987;106(3): 179-181.

15. Madan S, Blakeway C. Radiation exposure to surgeon and patient in intramedullary nailing of the lower limb. Injury 2002; 33(8):723-727.

16. Mehlman CT, DiPasquale TG. Radiation exposure to the orthopaedic surgical team during fluoroscopy: How far away is far enough? J Orthop Trauma 1997;11(6):392-398.

17. Toivanen JA, Vaisto O, Kannus P, Latvala K, Honkonen SE, Jarvinen MJ. Anterior knee pain after intramedullary nailing of fractures of the tibial shaft. A prospective, randomized study comparing two different nail-insertion techniques. J Bone Joint Surg Am 2002;84-A(4):580-585.

18. Keating JF, Orfaly R, O’Brien PJ. Knee pain after tibial nailing. J Orthop Trauma 1997;11(1):10-13.

19. Finkemeier CG, Schmidt AH, Kyle RF, Templeman DC, Varecka TF. A prospective, randomized study of intramedullary nails inserted with and without reaming for the treatment of open and closed fractures of the tibial shaft. J Orthop Trauma 2000;14(3): 187-193. 